https://helda.helsinki.fi

\title{
Empathizers and systemizers process social information differently
}

Riekki, Tapani

2018

Riekki , T , Svedholm-Häkkinen , A M \& Lindeman , M 2018 , ' Empathizers and systemizers process social information differently ' , Social Neuroscience , vol. 13 , no. 5 , pp. 616-627 . https://doi.org/10.1080/1

http://hdl.handle.net/10138/310989

https://doi.org/10.1080/17470919.2017.1368700

unspecified

acceptedVersion

Downloaded from Helda, University of Helsinki institutional repository.

This is an electronic reprint of the original article.

This reprint may differ from the original in pagination and typographic detail.

Please cite the original version. 
Running head: EQ/SQ and social information processing

\section{Empathizers and systemizers process social information differently}

Tapani Riekki*, Annika Svedholm-Häkkinena, and Marjaana Lindeman ${ }^{\mathrm{a}}$

Author Note

*Corresponding author: Department of Psychology, Faculty of Medicine, University of Helsinki, P.O. Box 9, 00014 University of Helsinki, Finland. Phone number: +358 440424281, E-mail: tapani.riekki@helsinki.fi, fax: +3580294129542

${ }^{a}$ Department of Psychology, Faculty of Medicine, University of Helsinki Helsinki. P.O. Box 9, 00014 Finland.

Annika Svedholm-Häkkinen: annika.svedholm@helsinki, +358-2941 29404

Marjaana Lindeman: marjaana.lindeman@helsinki.fi, +358-2941 29402

\section{Acknowledgements}

This study was supported by the Research Funds of the Academy of Finland under Grant No. 265518.

Word count: $\underline{5232}$ 


\section{Abstract}

Using the empathizing-systemizing theory as our framework, we investigated how people with high selfreported empathizing (having good social skills and being interested in people) and systemizing (being interested in physical things and processes) differ in the social information processing of emotionally negative photographs of people during "spontaneous watching" and emotional and cognitive empathy tasks. Empathizers evaluated the pictures as more emotionally touching and the reactions in the photographs more understandable than the systemizers. Compared to the empathizers, systemizers had stronger activations in the posterior cingulate cortex, an area related to cognitive empathy, as well as in the left superior temporal gyrus and middle frontal gyrus when watching emotional photographs spontaneously. During guided emotional and cognitive empathy tasks, these differences disappeared. However, during the emotional empathy task, higher systemizing was associated with weaker activation of the right inferior frontal gyrus / insula. Furthermore, during emotional and cognitive empathy tasks, empathizing was related to increased activations of the amygdala which were in turn related to higher behavioral ratings of emotional and cognitive empathy. The results suggest that empathizers and systemizers engage in social information processing differently: systemizers in more cognitive terms and empathizers with stronger automatic emotional reactions.

Keywords: cognitive empathy, emotional empathy, systemizing, empathizing, fMRI 


\section{Introduction}

For more than 100 years, the different ways in which people process social and physical information have been considered to be the most fundamental individual differences in human interests and abilities (a review: Su, Rounds, \& Armstrong, 2009). One of the theories considering these differences is BaronCohen's Empathizing-Systemizing (E-S) theory (Baron-Cohen, 2009; Baron-Cohen, Knickmeyer, \& Belmonte, 2005). According to E-S theory, empathizing and systemizing traits are normally distributed throughout the population. Strong empathizers are good at understanding the social world; they are people who are good at emotion recognition and social sensitivity, who value developing altruistic relationships, who are interested in fiction and people, and who often have female-typed hobbies and occupations in such fields as humanities or social care. Strong systemizers, in turn, are good at understanding the physical world, at how things work; they have high constructional abilities and are detail-oriented, they are good at spatial navigation, map reading, and problems of physics, and they often choose occupations linked to lawful phenomena in the material world, such as engineering, computers, and mathematics (Baron-Cohen, 2002; Baron-Cohen et al., 2005). In the present study, we will examine how strong empathizers and strong systemizers differ in social information processing and what are the brain basis of these differences.

Like other theories (e.g., Cox et al., 2012; Davis, 1983; Decety \& Jackson, 2004), the E-S theory sees empathy as consisting of a cognitive component (the ability to, and interest in, understanding what other people are thinking and feeling) and an emotional component (feeling for another and sharing another's emotional state). Although several questions remain, this division of empathy into two separate components is supported by brain imaging studies, which have identified two networks, the cognitive and emotional empathy networks (Fan, Duncan, de Greck, \& Northoff, 2011; Van Overwalle, 2011; Van Overwalle \& Baetens, 2009; Schurz, Radua, Aichhorn, Richlan, \& Perner, 2014; Shamay-Tsoory, AharonPeretz, \& Perry, 2009). The cognitive empathy network (sometimes referred to as the mentalizing network) often includes areas in the temporoparietal junction/superior temporal gyrus (TPJ/STG), medial prefrontal 
cortex (mPFC) including sometimes parts of the anterior cingulate cortex (ACC), and precuneus (PCC). Tasks that often activate these areas include tasks where participants must predict or understand other people's mental states, points of view, and thinking, such as false belief tasks and story comprehension tasks. The emotional empathy network is usually associated with areas in the anterior intraparietal sulcus, premotor cortex, middle cingulum, and insula, and recently also the amygdala (Bruneau, Jacoby, \& Saxe, 2015). Usually tasks that activate areas associated with emotional empathy are related to processing of others' suffering and pain, mirroring of emotional states, and imitation. Because the research on social neuroscience can be considered fragmented with different tasks, methods, and theory, Alcalá-López and colleagues (2017) examined with meta-analytic connectivity modeling the different neural processing levels related to social skills and social-cognitive capacities. They identified four different processing levels, lower sensory, limbic, intermediate, and high associate neural circuits that contribute to different levels of processing of social information and none of which are uniquely domain-specific to social information processing. Thus, it seems that although the dichotomy of cognitive/emotional empathy has its place, overall, the social information processing is a complex phenomenon. Despite this complexity, in this paper, we focus especially on activations related to areas commonly associated with emotional and cognitive empathy.

Besides empathizing and systemizing, the E-S theory has launched the concept of "brain type". Individuals in whom self-evaluated systemizing is at a higher level than their empathizing are said to have a systemizing "brain type" ("systemizers"), and individuals in whom empathizing is at a higher level than their systemizing are said to have an empathizing "brain type" ("empathizers") (Wakabayashi, Baron-Cohen, \& Ashwin, 2012). Empirical tests of the predictions that empathizing, systemizing, and the "brain type" have wider-ranging effects on healthy people's cognition, interests and hobbies, as well as success in social interactions and technology, have started to accumulate, and the results have largely been in support of the theory (Nettle, 2007; Svedholm-Häkkinen \& Lindeman, 2016; Wright, Eaton, \& Skagerberg, 2015; Zeyer et al., 2013). E-S theory suggest that empathizing and systemizing may "compete in the brain" (Baron- 
Cohen, et al., 2003). However, studies show that while the two traits are strongly negatively correlated in clinical ASD populations (Grove, et al., 2013; Wheelwright et al., 2006), in nonclinical populations, they show only a small negative association (Grove, et al., 2013; Nettle, 2007; Wheelwright et al., 2006). Nevertheless, as the theory focuses on the relative difference between empathizing and systemizing, we too focused our analyses on this difference in addition to the possible independent relations of empathizing and systemizing to brain activations.

Although systemizing has been associated with structural differences in areas related to cognitive control, monitoring, error detection, and probabilistic inference (Lai et al, 2012), the way systemizers and empathizers differ in the processing of social information, especially the brain correlates of those differences, is largely unknown. One particularly interesting question is whether the empathetic reactions of strong systemizers are simply weaker than empathizers' reactions. More specifically, do areas of the brain related to emotional empathy activate less in systemizers than in empathizers when seeing people in distress or other empathy-eliciting situations? Another possibility is that systemizers process social information qualitatively differently from empathizers. Since systemizing behaviors entail an analytic stance and a search for rules, this begs the question: Do strong systemizers process social material in a more cognitive manner than others? If they do, is this reflected in their brain activations, specifically in those areas associated with the cognitive empathy network?

These are the main questions that this study addressed. We examined how activation of areas of the brain that are related to the emotional and cognitive empathy networks, together with other possible activations, differs between systemizers, empathizers, during three tasks, as well as how these activations are related to behavioral ratings of stimuli. Our study questions are thus explorative, because clear hypotheses are hard to draw from earlier research. To our knowledge there have been only two studies conducted on healthy populations that have studied these issues, with mixed results and methods (Focquaert, Steven-Wheeler, Vanneste, Doron, and Platek, 2010; Kestemont, Vandekerckhove, Bulnes, 
Matthys and Overwalle, 2016). The first task, spontaneous watching, was without explicit instructions since explicit instructions are known to have effects on social information processing (Baron-Cohen, 2002) and because spontaneous reactions to social stimuli have been studied surprisingly little (Wagner, Kelley, \& Heatherton, 2011). The second task was the emotional empathy task, where we instructed participants to focus on the emotional quality of photographs of people that were either emotional or neutral. In the third task, the cognitive empathy task, participants were instructed to focus on the understandability of the person's mental state in the same photographs.

\section{Materials and methods}

\section{Participants}

We recruited 38 healthy (no psychiatric or neuropsychiatric disorders) volunteers (mean age 31, range 2046 years, $50 \%$ female) from a larger population of participants in an earlier study on empathizing, systemizing, thinking and beliefs. Following Goldenfield, Baron-Cohen, and Wheelwright (2005), we calculated the difference score between standardized scores on the 15-item Empathy Quotient scale (EQ; Muncer \& Ling, 2006) and the scores on the 18-item Systemizing Quotient scale (SQ; Ling, Burton, Salt, \& Muncer, 2009). In order to recruit participants with an empathizing or systemizing cognitive profile, only those whose difference score was among the upper or lower $25 \%$ for their gender were invited to the present study. Participants were not matched on demographic characteristics. In the main analyses, we used the participants' scores on this continuous variable. For ease of expression, we will refer to low scores on this variable as "empathizing brain type" and to high scores as "systemizing brain type". Because the "brain type" is self-rated, not a brain measurement, and to avoid confusing it with our actual brain imaging variables, we will refer to it in quotation marks throughout the text. The overall mean of the "brain type" score in the whole sample was $3.3(S D=23.28$; range: $-31.14-42.14)$, for men $4.3(S D=20.57$; range: $31.14-33.06)$, and for women $2.31(S D=-26.25$; range: $-30.33-42.14)$. 
We used 25 emotional pictures of people, 25 neutral pictures of people, and 25 pictures of things.

Emotional pictures depicted people in distress, who were suffering from pain, injury, grief, or other negative emotions, and were taken from the Pictorial Empathy Test and its pilot study (Lindeman, Koirikivi, \& Lipsanen, 2016). Rest of the pictures were taken from Wikimedia Commons. Neutral pictures were profile pictures of people looking at ease and the pictures of things depicted lifeless devices such as machines (e.g., a clock) and tools (e.g., a screwdriver).

\section{Procedure}

Prior to entering the $\mathrm{fMRI}$ scanner, participants practiced answering questions with a visual analog scale (VAS), used later in the empathy tasks. One practice picture from each category of the stimuli was shown, followed by the question "How nice is the picture?". The participants were told that there were no right or wrong answers and they were encouraged to answer based on how they felt. After the practice questions were completed, participants entered the scanner to perform the following four tasks, always presented to participants in the same order. There were no breaks between the tasks except for the time that it took to read the instructions for the next task.

In the first task, spontaneous watching, all 75 pictures were presented to participants in random order; no explicit instructions were given, participants were simply told to watch. A trial consisted of a fixation cross $(1000 \mathrm{~ms})$ and a picture $(3000 \mathrm{~ms})$, with a random length break $(1000-1500 \mathrm{~ms})$ of an empty screen between trials. After this task, there was a short break and participants were checked on before continuing to the next task.

The second task was an emotional empathy task. The 25 emotional and 25 neutral pictures of people were presented in random order and participants were instructed to "watch the pictures again and rate how emotionally touching the pictures are". A trial consisted of a fixation cross (random length of $1500-2000 \mathrm{~ms}$ ) following by a picture (3000ms). After the picture presentation, the question "How emotionally touching 
was the picture?" was presented in the middle of the screen along with a VAS (left side: $-250=$ not emotionally touching; right side: $250=$ emotionally touching). Answers were given by moving a cursor along the VAS with two buttons. Maximum answering time was $8000 \mathrm{~ms}$ (all participants answered inside this time window in all phases).

The third task was a things evaluation task. The presentation of the 25 pictures of things was similar to the second task. The instruction was to consider "How interesting is the machine/tool and how could it work" and the question and the response options were changed to "How interesting was the machine/tool" (-250 $=$ uninteresting, $250=$ interesting) .

The fourth task was a cognitive empathy task. All 25 emotional and 25 neutral pictures of people were presented to the participants as in the second task, but the question and response options were changed to "How understandable are the expressions of the person in the picture?" $(-250=$ hard to understand, $250=$ easy to understand).

\section{fMRI parameters and analyses}

Imaging was done with a MAGNETOM Skyra 3.0 T (Siemens, Erlangen) scanner. Parameters were: echo time $32 \mathrm{~ms}$, repetition time $1.5 \mathrm{~s}$, flip angle $75^{\circ}, 36$ slices aligned with the line connecting the anterior and posterior commissures, slice thickness $4.0 \mathrm{~mm}$ and matrix size $64 \times 64$. Analyses were done with SPM12 software. Functional images were realigned to the same space via linear rotation and were then translated to correct for movement, then normalized, and, finally, smoothed (full width at half maximum $=8 \mathrm{~mm}$ ).

Functional time series were analyzed using a general linear model. In the spontaneous watching task, boxcar functions were entered for different pictures (emotional, neutral, and things), and fixation cross. In the emotional empathy task, the things evaluation task, and the cognitive empathy task, box-car functions for pictures (emotional, neutral, and things) as well as answering and fixation cross were used. Movement 
parameters were entered as confounding covariates in cases where movements larger than half a voxel occurred during the scanning. For the spontaneous watching task, the fMRI data were fitted to the model and individual contrast images were calculated for group-level statistical tests.

For the cognitive and emotional empathy tasks, and the things evaluation task, we used a $2 \times 3$ flexible full factorial design where the task (cognitive/emotional empathy) and stimuli type (emotional/neutral people) were modeled with two levels. The things evaluation task was modeled with one level because it had only one type of stimuli and one task. We first calculated the main effects for task (emotional/cognitive empathy) and stimuli type (emotional people/neutral people/things), and the possible two-way interaction effects of the empathy tasks and people stimuli types. Then, we used t-tests for pairwise comparisons to further study the effects of individual contrasts. Comparisons to neutral people from both, the spontaneous watching task, and from the emotional and cognitive empathy tasks can be found in the supplementary materials.

We modeled the continuous variable of empathizing/systemizing "brain type" as a regressor (technically covariate in the SPM) in the SPM models. Similarly, we also used its components, the EQ score, and the SQ score in two separate models in SPM to further clarify the possible driving forces of the found activations. In other words, in all comparisons we ran analyses with three different regressions: empathizing/systemizing "brain type", only the EQ score, and only the SQ score. The empathizing/systemizing "brain type", the EQ score, and the SQ score were analyzed in separate models because of their multicollinearity.

Statistical thresholds were set in two ways. The first statistical threshold comprised at least 10 voxels exceeding voxel-wise $p<0.001$ (uncorrected) corrected for multiple comparisons at the voxel-level (peaklevel) to the entire brain volume (FWE $=0.05)$. Second, we used a region of interest $(\mathrm{ROI})$ analysis with one continuous ROI that was taken from Spunt and Adolph's (2014) why/how localizer task that taps especially 
on cognitive empathy. One common ROI-mask was generated and used for all analyses in the present study. ROI analysis was conducted with the same statistical thresholds (FWE $=0.05)$ as in the general analysis except that the multiple comparisons were constricted to the volume of the ROI. The ROI included areas of the $\mathrm{mPFC}$, the inferior frontal areas, the temporal areas (temporal pole and middle temporal gyrus), the superior temporal gyrus areas (including the TPJ), and the PCC. We used voxel-wise corrections due to recent findings showing that cluster-wise corrections may inflate the statistical significance of results (Eklund, Nichols, \& Knutsson, 2016). We also extracted the average signal change of the amygdala using an anatomical ROI that was used in correlation analysis with the behavioral variables.

\section{Results}

\section{Behavioral results}

Correlations between the systemizing/empathizing "brain type", EQ and SQ scores, and evaluation task scores are presented in Table 1. The empathizers rated the emotional pictures as being both more emotionally touching and more understandable than systemizers. EQ scores correlated with rating the emotional pictures as more understandable while SQ scores correlated with finding the reactions in the emotional pictures to be less understandable.

\section{Spontaneous watching task}

Figure 1 shows the brain activations in the emotional people > things comparison. In the emotional people $>$ things comparison, there were activation clusters in areas associated with the cognitive empathy network (bilateral STG, MPFC, but not PCC), right middle temporal gyrus, and an activation cluster in visual areas (Table 2). The inverse comparison (things > people) revealed stronger activations for things in the bilateral fusiform gyrus, bilateral occipital areas, and in the left inferior parietal lobule. Average amygdala activations extracted from the anatomical ROI during spontaneous watching were neither related to SQ or EQ score nor systemizing/empathizing "brain type". However, activation of the right amygdala during spontaneous 
watching of the emotional pictures did correlate with participants' behavioral evaluations of how emotionally touching the emotional pictures, seen later during the emotional empathy task, were $(r=.36, p$ $=.027)$. For the emotional people $>$ neutral people comparison, please see the supplementary materials.

In the regression analyses of emotional people > things comparison (Table 2, Figure 1), systemizing "brain type" was associated with activation clusters in the PCC and the left STG. Furthermore, the activation of the peak-voxel of the PCC cluster correlated negatively with behavioral evaluations regarding how emotionally touching the emotional pictures were that were given later during the emotional empathy task $(r=-.37, \mathrm{p}=$ .023). Similarly, high SQ scores were associated with activations in the PCC and left STG as well as an activation cluster in the right middle frontal gyrus extending toward the mPFC. This shows that there was more activation in areas related to cognitive empathy among systemizers than empathizers. The peak-voxel activations of the PCC and middle frontal gyrus were inversely associated with later behavioral ratings of how emotionally touching the emotional pictures were (PCC: $r=-.50, p=.001$; middle frontal gyrus: $r=-$ $.35, p=.03)$. Analysis done with the EQ score did not reveal any associations.

\section{Main effects of the emotional and cognitive empathy tasks}

Main effects for the task (cognitive/emotional empathy) are presented in Table 3 and Figure 2. Main activation differences were in the left superior temporal gyrus, right precentral gyrus, left fusiform gyrus, and in a large activation cluster in the right basal ganglia that spread towards the insula and the IFG. There was also a large activation cluster in the bilateral supplementary motor area that included parts of the middle cingulum. In the pairwise comparison between the emotional empathy > cognitive empathy tasks (emotional + neutral pictures during the emotional empathy task $>$ the emotional + neutral pictures during the cognitive empathy task), there was an activation cluster in the right basal ganglia / IFG, showing stronger activation to the emotional empathy task (Table 4.). The reverse comparison did not reveal any activations. Regression analysis did not reveal associations with empathizing/systemizing "brain type", the EQ score, nor the SQ score. 
Main effects of the emotional and neutral people stimuli

Main effects for the stimuli type of emotional/neutral people are presented in Table 3. A large bilateral activation cluster was found in the occipital areas spreading towards superior temporal gyri and ventral precuneus. There were also activation clusters in the right IFG, mPFC, PCC, and in the left precentral gyrus/IFG. A pairwise comparison between emotional people $>$ neutral people (emotional people during cognitive and emotional empathy tasks > neutral people during cognitive and emotional empathy tasks) showed stronger activations for emotional people in the occipital and temporal areas, left IFG, right Thalamus, left middle frontal gyrus/precentral gyrus, and in the right IFG (Supplementary Table 2). Regression analysis did not reveal associations with empathizing/systemizing "brain type", the EQ score, nor the SQ score.

Main effects of the things evaluation task

Main effects for the things evaluation task are presented in Table 3. Large bilateral activation clusters were found in the occipital areas spreading towards inferior parietal lobule and towards calcarine and in PCC, mPFC, and bilateral precentral gyri. Regression analysis did not reveal associations with empathizing/systemizing "brain type", the EQ score, nor the SQ score.

Interaction of the cognitive and emotional tasks and the emotional and neutral people An interaction effect (Figure 2, Table 3) of task and stimuli was found in the right basal ganglia/IFG showing stronger activation in this area for emotional people stimuli during the emotional empathy task (Table 4). Regression analysis did not reveal associations with empathizing/systemizing "brain type", the EQ score, nor the SQ score.

Pairwise comparisons of the emotional people stimuli during the emotional/cognitive empathy tasks and the things evaluation task 
Results from the pairwise comparison between emotional people during emotional empathy task > things evaluation task are presented in Table 4 (for the inverse comparison and emotional people during cognitive empathy task > things evaluation task comparison, please see the supplementary materials). In the regression analyses, high SQ scores were associated with weaker activations of the right IFG / insula (Figure 2, Table 4). This right IFG / insula cluster is hard to interpret as it is mostly located in white matter and covers parts of the IFG (151 voxels), insula (42 voxels), and caudate (49 voxels). However, the peak-voxel activation of the cluster correlated negatively with the behavioral ratings of how emotionally touching the emotional pictures were $(r=-.33, p=.041)$ connecting the finding to emotional empathy. Additionally, in the emotional people stimuli during emotional empathy task > things evaluation task comparison, first, higher activations of the left $(r=.40, p=.012)$ and the right $(r=.34, p=.037)$ amygdala were associated with behavioral ratings of how emotionally touching the emotional pictures were. Second, higher left amygdala activations were associated with empathizing "brain type" (left: $r=.43, p=.008$; right: $r=.30, p$ $=.068)$ and to higher EQ scores $(r=.41, p=.013)$. These results show that empathizers in contrast to systemizers had stronger activation in areas related to emotional empathy. Furthermore, in the emotional people during the cognitive empathy task > things evaluation task comparison, higher left amygdala activations were associated with rating the people's reactions in the pictures as more understandable $(r=$ $.37, \mathrm{p}=.023)$ and with empathizing "brain type" $(r=.36, p=.027)$. High SQ score, on the other hand, was inversely associated with right amygdala activation $(r=-.41, p=.011)$.

\section{Discussion}

In the current study, we were interested in whether systemizers and empathizers process social information differently and what the brain correlates of these differences are. The results are among the first to show that there is less activation in areas related to emotional empathy processing among systemizers when seeing people in distress, and that systemizers used areas related to cognitive empathy more often than empathizers during spontaneous situations. 
When participants were watching emotionally negative pictures without instructions, systemizing (both SQ score alone and the systemizing "brain type") was associated with activations related to cognitive empathy, namely the PCC and the left STG. In addition, high SQ scores were related to activations in the middle frontal gyrus. However, no differences between empathizers and systemizers were found regarding areas related to emotional empathy or the amygdala during spontaneous watching. The PCC is typically associated with conscious cognitive empathy processing, such as judging false beliefs and making trait judgments (Schurz et al., 2014), and together with the left temporal areas and middle frontal gyrus, is associated to threat assessment (Fiddick, 2011; Qin \& Han, 2009; Qin, Lee, Wang, Mao, \& Han, 2009). The middle frontal areas, on the other hand, have been associated with explicit down-regulation of negative emotions (Bruneau et al., 2015; Ochsner et al., 2004; Ochsner \& Gross, 2005). Together, the activations of the PCC, left STG, middle temporal gyrus and middle frontal gyrus suggest that systemizers do not simply evince weaker emotional empathy processing than empathizers in spontaneous situations. Rather, systemizers may engage in more cognitive empathy processing during spontaneous situations than empathizers.

Our finding that systemizers had stronger activations in areas related to cognitive empathy (PCC, STG) is surprising. Our behavioral results from the emotional and cognitive empathy tasks showed that people high in systemizing evaluated the pictures of people in distress as emotionally less moving and less understandable than people with high empathizing- a finding that is in line with the basic tenet of the E-S theory, that people with systemizing "brain type" have weaker empathy than people with empathizing "brain type" (Baron-Cohen, 2009). What could this discrepancy between fMRI data and behavioral results mean? Brain research related to the E-S theory has mostly been performed on people with autism spectrum disorders (ASD) and to our knowledge, only two fMRI studies regarding social information processing and the E-S theory have been done using the general population. Focquaert, Steven-Wheeler, Vanneste, Doron, and Platek (2010) found, using empathizing/systemizing "brain type" as the 
measurement, that during an explicit face-based mindreading task, empathizers had stronger activations than systemizers in emotional empathy-related areas and autobiographical / perspective-taking areas, while systemizers had stronger activations in the parahippocampal gyrus, the left STG, and the left IFG. Our findings from the spontaneous watching task showing extraneural activity for the higher systemizing especially in the left STG, would fit with this finding, although we did not find differences regarding emotional empathy activations during the spontaneous watching task. Kestemont, Vandekerckhove, Bulnes, Matthys and Overwalle (2016), on the other hand, found that a sub-clinical ASD group had more extensive activations in several brain areas, including the left STG, than the controls during a reading-based social information task. Based on their finding, Kestemont and colleagues suggested that the extraneural activity among the sub-clinical ASD group might reflect use of compensatory strategies and extra resources during the task. Our findings regarding associations of activations of cognitive empathy areas with the systemizing, together with frontal activations associating with high SQ scores, would fit with this interpretation: the task may have been more laborious for people with higher systemizing than to people with higher empathizing, leading the systemizers to think through the situation explicitly and analytically with a cognitive stance.

When the participants were instructed to focus either on how emotionally touching the pictures were (emotional empathy task) or how understandable the reactions in the pictures were (cognitive empathy task), differences in the cognitive empathy area activations between systemizers and empathizers vanished. However, new differences were found relating to emotional empathy. In the emotional empathy task, people with low SQ scores had a stronger activation than people with high SQ scores in a cluster in the right IFG / insula. Furthermore, empathizing "brain type", together with higher EQ scores, were related to both higher amygdala activations and to reporting the distressing pictures as more emotionally touching. Similar associations for amygdala activations were also found in the cognitive empathy task where it was associated with both empathizing "brain type", lower SQ scores, and to rating the reactions in the pictures as more understandable. Both the right IFG and the insula have been associated with emotional empathy 
(Lamm \& Singer, 2010; Shamay-Tsoory et al., 2009) and a recent study showed that the amygdala may contribute to other-focused empathy as well (Bruneau et al., 2015). It is possible that these activations do not reflect genuine empathy (or sympathy) but more general emotional reactions to content of the pictures because the instruction of the task ("How emotionally touching is the picture") can be considered ambiguous to some extent. Furthermore, because these activations were only found in in pairwise comparisons between the emotional pictures stimuli during the emotional empathy tasks and the things evaluation task, it is also possible that these differences are at least partly driven by different reactions to things. Nevertheless, these findings suggest that under explicit appeal to use emotional empathy, empathizers may engage in more emotional empathy than systemizers. However, the findings also imply that even under conditions that encourage cognitive empathy, empathizers engage with more emotional empathy than systemizers. This is in line with a finding showing that during explicit face-based mindreading task, empathizers show stronger activations related to emotional empathy (Focquert et al., 2010). In the future, these findings may serve as a foundation for further examination of the interplay between emotional and cognitive empathy, especially among strong empathizers.

\section{Potential limitations}

Most of the differences between empathizers and systemizers were found in the emotional people $>$ things comparison, thus, it may be that these differences stem from different reactions to things (tools, machines) rather than pictures of emotional people. However, two things counter this interpretation. First, if the differences were mostly driven by different activations related to things, these differences should be also observable in the neutral people $>$ things comparisons. We analyzed the neutral people $>$ things comparisons and found no differences between systemizers and empathizers. Second, there were several similar, but statistically non-significant, activations observable in the emotional people $>$ neutral people contrasts, as in the emotional people > things contrasts. Thus, although using things in the comparison may have affected the effect sizes, the effects seem to be mostly related to different reactions to emotional 
pictures of people. Another limitation is that the PCC and the left STG are not unambiguous manifestations of cognitive empathy, since the PCC and left STG are not only related to social information processing but to the default mode network (DMN; Raichle, 2015), activation of which is considered to reflect detachment from stimuli-driven tasks. This raises the question of whether our activations reflected detachment from the task, which in turn, would explain the behavioral findings of systemizers rating the pictures as less touching and less understandable than empathizers. However, because activations were also found outside the areas that are usually linked to the DMN and the same DMN-related areas activated in the cognitive/emotional empathy tasks, it is more likely that these activations were not related to taskdisengagement but to cognitive empathy. It should also be noted that we used the same pictures repeatedly in different tasks. The strength of this approach is that the differences between the task activations were likely related to the different tasks and not to the stimulus material. However, because seeing the same pictures, of for example emotional people, three times may have had effects on the activations, this should be controlled for in future studies, and possible interactions of the "brain type" and the repeated use of same stimulus material could be also studied. In a similar manner, because the task order was the same for all participants, we were not able to control for different strategies that systemizers/empathizers may have used and this could be also controlled for in future studies. Finally, our participants were healthy people without psychiatric or neuropsychiatric diagnoses. However, because for example depressive mood can affect social information processing, in future studies some clinical measurements and assessments could be used to clarify the results.

\section{Conclusions and implications for the E-S theory}

In the present study, we found that participants' self-rated empathizing and systemizing tendencies were reflected in different brain activations while they processed photographs of people in distress. The results suggest that when spontaneously observing other people's suffering, systemizers may take a more cognitive stance than empathizers. Additionally, during more focused observations, systemizers utilize emotional empathy less than empathizers, reflected in the differences in the amygdala and right IFG / 
insula activations. The results extend the E-S theory in important ways. The theory suggests that our tendencies toward empathizing or systemizing have wide-ranging implications for our interests, hobbies, occupations, and performance on a range of everyday tasks (Baron-Cohen, 2002; Baron-Cohen et al., 2005). Our results show that empathizing and systemizing are also linked to differences in the recruitment of either cognitive or emotional empathy networks during social information processing. In other words, not only empathizing, but also systemizing, plays an important role in the qualitative ways people relate to the social world. Thus, when measuring individual differences in social cognition, especially at the brain level, among healthy participants, not only should tendencies toward empathizing traits be used, but attention should be paid to systemizing traits as well. In summary, the results suggest that for some of us, understanding other people's situations and reacting to others' suffering is easy, maybe even intuitively automatic, while for others, these tasks may require a more cognitive stance. 


\section{References}

Alcalá-López, D., Smallwood, J., Jefferies, E., Van Overwalle, F., Vogeley, K., Mars, R. B., ... \& Bzdok, D. (2017). Computing the Social Brain Connectome Across Systems and States. Cerebral Cortex, 1-26. doi: 10.1093/cercor/bhx121

Baron-Cohen, S. (2002). The extreme male brain theory of autism. Trends in Cognitive Sciences, 6, 248- 254. doi:10.1016/S1364-6613(02)01904-6

Baron-Cohen, S. (2009). Autism: The Empathizing-Systemizing (ES) Theory. Annals of the New York Academy of Sciences, 1156, 68-80. doi:10.1111/j.1749-6632.2009.04467.x

Baron-Cohen, S., Knickmeyer, R. C., \& Belmonte, M. K. (2005). Sex differences in the brain: Implications for explaining autism. Science, 310, 819-823. doi:10.1126/science.1115455

Baron-Cohen, S., Richler, J., Bisarya, D., Gurunathan, N., \& Wheelwright, S. (2003). The systemizing quotient: an investigation of adults with Asperger syndrome or figh-functioning autism, and normal sex differences. Philosophical Transactions of the Royal Society of London. Series B, Biological Sciences, 358, 361-374. doi: $10.1098 /$ rstb.2002.1206

Bruneau, E. G., Jacoby, N., \& Saxe, R. (2015). Empathic control through coordinated interaction of amygdala, theory of mind and extended pain matrix brain regions. Neurolmage, 114, 105-119. doi:10.1016/j.neuroimage.2015.04.034

Caldwell-Harris, C. L., \& Jordan, C. J. (2014). Systemizing and special interests: Characterizing the continuum from neurotypical to autism spectrum disorder. Learning and Individual Differences, 29, 98-105. doi:10.1016/j.lindif.2013.10.005

Cox, C. L., Uddin, L. Q., Di Martino, A., Castellanos, F. X., Milham, M. P., \& Kelly, C. (2012). The balance between feeling and knowing: Affective and cognitive empathy are reflected in the brain's intrinsic functional dynamics. Social Cognitive and Affective Neuroscience, 7, 727-737. doi:10.1093/scan/nsr051 Davis, M. H. (1983). Measuring individual differences in empathy: Evidence for a multidimensional approach. Journal of Personality and Social Psychology, 44, 113-125. doi:10.1037/0022-3514.44.1.113 
Decety, J., \& Jackson, P. L. (2004). The functional architecture of human empathy. Behavioral and Cognitive Neuroscience Reviews, 3, 71-100. doi:10.1177/1534582304267187

Eklund, A., Nichols, T. E., \& Knutsson, H. (2016). Cluster failure: Why fMRI inferences for spatial extent have inflated false-positive rates. Proceedings of the National Academy of Sciences, 201602413. doi:10.1073/pnas.1602413113

Goldenfield, N., Baron-Cohen, S., \& Wheelwright, S. (2005). Empathizing and systemizing in males, females, and autism. Clinical Neuropsychiatry, 2, 338-345.

Grove, R., Baillie, A., Allison, C., Baron-Cohen, S., \& Hoekstra, R. A. (2013). Empathizing, systemizing, and autistic traits: latent structure in individuals with autism, their parents, and general population controls. Journal of Abnormal Psychology, 122, 600-609. doi: 10.1037/a0031919

Fan, Y., Duncan, N. W., de Greck, M., \& Northoff, G. (2011). Is there a core neural network in empathy? An fMRI based quantitative meta-analysis. Neuroscience \& Biobehavioral Reviews, 35, 903-911. doi:10.1016/j.neubiorev.2010.10.009

Fiddick, L. (2011). There is more than the amygdala: Potential threat assessment in the cingulate cortex. Neuroscience \& Biobehavioral Reviews, 35, 1007-1018. doi:10.1016/j.neubiorev.2010.09.014 Focquaert, F., Steven-Wheeler, M. S., Vanneste, S., Doron, K. W., \& Platek, S. M. (2010). Mindreading in individuals with an empathizing versus systemizing cognitive style: An fMRI study. Brain Research Bulletin, 83, 214-222. doi:10.1016/j.brainresbull.2010.08.008

Kestemont, J., Vandekerckhove, M., Bulnes, L. C., Matthys, F., \& Van Overwalle, F. (2015). Causal attribution in individuals with subclinical and clinical autism spectrum disorder: An fMRI study. Social Neuroscience, 11, 264-276. doi:10.1080/17470919.2015.1074104

Lamm, C., \& Singer, T. (2010). The role of anterior insular cortex in social emotions. Brain Structure and Function, 214, 579-591. doi:10.1007/s00429-010-0251-3

Lindeman, M., Koirikivi, I. \& Lipsanen, J. (2016). Pictorial Empathy Test (PET). An easy-to-use method for assessing affective empathic reactions. European Journal of Psychological Assessment. doi:10.1027/1015$5759 / a 000353$ 
Lindeman, M., Svedholm-Häkkinen, A. M., \& Lipsanen, J. (2015). Ontological confusions but not mentalizing abilities predict religious belief, paranormal belief, and belief in supernatural purpose. Cognition, 134, 6376. doi:10.1016/j.cognition.2014.09.008

Ling, J., Burton, T. C., Salt, J. L., \& Muncer, S. J. (2009). Psychometric analysis of the systemizing quotient (SQ) scale. British Journal of Psychology, 100, 539-552. doi:10.1348/000712608X368261

Muncer, S. J., \& Ling, J. (2006). Psychometric analysis of the empathy quotient (EQ) scale. Personality and Individual Differences, 40, 1111-1119. doi:10.1016/j.paid.2005.09.020

Nettle, D. (2007). Empathizing and systemizing: What are they, and what do they contribute to our understanding of psychological sex differences? British Journal of Psychology, 98, 237-255. doi:10.1348/000712606X117612

Ochsner, K. N., \& Gross, J. J. (2005). The cognitive control of emotion. Trends in Cognitive Sciences, 9, 242249. doi:10.1016/j.tics.2005.03.010

Ochsner, K. N., Ray, R. D., Cooper, J. C., Robertson, E. R., Chopra, S., Gabrieli, J. D., \& Gross, J. J. (2004). For better or for worse: neural systems supporting the cognitive down-and up-regulation of negative emotion. Neuroimage, 23, 483-499. doi:10.1016/j.neuroimage.2004.06.030

Qin, J., \& Han, S. (2009). Neurocognitive mechanisms underlying identification of environmental risks. Neuropsychologia, 47, 397-405. doi:10.1016/j.neuropsychologia.2008.09.010

Qin, J., Lee, T. M., Wang, F., Mao, L., \& Han, S. (2009). Neural activities underlying environmental and personal risk identification tasks. Neuroscience Letters, 455, 110-115. doi:10.1016/j.neulet.2009.03.008

Raichle, M. E. (2015). The brain's default mode network. Annual Review of Neuroscience, 38, 433-447. doi:10.1146/annurev-neuro-071013-014030

Schurz, M., Radua, J., Aichhorn, M., Richlan, F., \& Perner, J. (2014). Fractionating theory of mind: A metaanalysis of functional brain imaging studies. Neuroscience \& Biobehavioral Reviews, 42, 9-34. doi:10.1016/j.neubiorev.2014.01.009 
Shamay-Tsoory, S. G., Aharon-Peretz, J., \& Perry, D. (2009). Two systems for empathy: A double dissociation between emotional and cognitive empathy in inferior frontal gyrus versus ventromedial prefrontal lesions. Brain, 132, 617-627. doi:10.1093/brain/awn279

Spunt, R. P., \& Adolphs, R. (2014). Validating the why/how contrast for functional MRI studies of theory of mind. Neuroimage, 99, 301-311. doi:10.1016/j.neuroimage.2014.05.023

Su, R., Rounds, J., \& Armstrong, P. I. (2009). Men and things, women and people: A meta-analysis of sex differences in interests. Psychological Bulletin, 135, 859-884. doi:10.1037/a0017364

Svedholm-Häkkinen, A., \& Lindeman, M. (2016). Testing the Empathizing-Systemizing theory in the general population: occupations, vocational interests, grades, hobbies, friendship quality, social intelligence, and sex role identity. Personality and Individual Differences, 90, 365-370. doi:10.1016/j.paid.2015.11.044

Van Overwalle, F. (2011). A dissociation between social mentalizing and general reasoning. Neuroimage, 54, 1589-1599. doi:10.1016/j.neuroimage.2010.09.043

Van Overwalle, F., \& Baetens, K. (2009). Understanding others' actions and goals by mirror and mentalizing systems: a meta-analysis. Neuroimage, 48, 564-584. doi:10.1016/j.neuroimage.2009.06.009

Wagner, D. D., Kelley, W. M., \& Heatherton, T. F. (2011). Individual differences in the spontaneous recruitment of brain regions supporting mental state understanding when viewing natural social scenes. Cerebral Cortex, 21, 2788-2796. doi:10.1093/cercor/bhr074

Wakabayashi, A., Baron-Cohen, S., \& Ashwin, C. (2012). Do the traits of autism-spectrum overlap with those of schizophrenia or obsessive-compulsive disorder in the general population? Research in Autism Spectrum Disorders, 6, 717-725. doi:10.1016/j.rasd.2011.09.008

Wheelwright, S., Baron-Cohen, S., Goldenfeld, N., Delaney, J., Fine, D., Smith, R., . . Wakabayashi, A. (2006). Predicting Autism Spectrum Quotient ( $\mathrm{AQ}$ ) from the Systemizing Quotient-Revised (SQ-R) and Empathy Quotient (EQ). Brain Research, 1079, 47-56. doi: 10.1016/j.brainres.2006.01.012

Wright, D. B., Eaton, A. A., \& Skagerberg, E. (2015). Occupational segregation and psychological gender differences: How empathizing and systemizing help explain the distribution of men and women into (some) occupations. Journal of Research in Personality, 54, 30-39. doi:10.1016/j.jrp.2014.06.004 
Zeyer, A., Çetin-Dindar, A., Md Zain, A. N., Juriševič, M., Devetak, I., \& Odermatt, F. (2013). Systemizing: A cross-cultural constant for motivation to learn science. Journal of Research in Science Teaching, 50, 10471067. doi:10.1002/tea.21101 
Table 1. Correlations between "brain types" (systemizers-empathizers), EQ scores, SQ scores, and the evaluation tasks.

123

1. "Brain type"

2. EQ score

$-.93 * *$

3. SQ score $.74 * * \quad-.53^{* *}$

4. Emotional empathy task $-.38^{*}$

.26 .16

5. Cognitive empathy task $-.40 *$ $.40 *$ $-.36^{*}$

Note: ${ }^{*} p<.05 ;{ }^{* *} p<.01$ 
Table 2. Activations during the spontaneous watching task's emotional people $>$ things comparison. Results for systemizing "brain type" and SQ score are from two separate SPM models where they were modeled as continuous covariates.

\begin{tabular}{|c|c|c|c|c|c|c|}
\hline Anatomical region & $x$ & y & $z$ & t-value & $\begin{array}{c}\mathrm{n} \text { of } \\
\text { voxels }\end{array}$ & p-value \\
\hline \multicolumn{7}{|c|}{ Emotional people $>$ things comparison } \\
\hline \multicolumn{7}{|l|}{ Superior temporal } \\
\hline gyrus (right) & 46 & -68 & 4 & 10.13 & 2418 & $>.000^{1}$ \\
\hline \multicolumn{7}{|l|}{ Superior/middle } \\
\hline temporal gyrus (left) & -48 & -78 & 6 & 9.12 & 1043 & $>.000^{1}$ \\
\hline Calcarine (left) & -10 & -98 & 18 & 6.60 & 709 & $.002^{1}$ \\
\hline mPFC & 6 & 54 & 28 & 6.03 & 847 & $.009^{1}$ \\
\hline \multirow{2}{*}{\multicolumn{7}{|c|}{ Emotional people > things comparison: systemizing "brain type" as a }} \\
\hline & & & & & & \\
\hline PCC & 2 & -52 & 24 & 4.57 & 796 & $.041^{2}$ \\
\hline STG (left) & -56 & -60 & 32 & 4.62 & 125 & $.038^{2}$ \\
\hline \multicolumn{7}{|c|}{ Emotional people > things comparison: High SQ score as a covariate } \\
\hline PCC & 0 & -48 & 20 & 5.7 & 1351 & $.022^{1}$ \\
\hline STG (left) & -58 & -56 & 36 & 5.40 & 252 & $.047^{1}$ \\
\hline Middle frontal gyrus & & & & & & $.050^{1}$ \\
\hline (right) & 44 & 22 & 48 & 5.37 & 286 & \\
\hline \multicolumn{7}{|c|}{ Things > emotional people comparison } \\
\hline Fusiform gyrus (left) & -26 & -50 & -12 & 8.23 & 351 & $>.000^{1}$ \\
\hline
\end{tabular}




\begin{tabular}{|c|c|c|c|c|c|c|}
\hline Cerebellum (left) & -26 & -38 & -24 & 6.81 & $*$ & $.001^{1}$ \\
\hline Fusiform gyrus (right) & 28 & -42 & -12 & 7.15 & 161 & $.001^{1}$ \\
\hline Cerebellum (right) & 24 & -40 & -20 & 6.77 & $*$ & $.001^{1}$ \\
\hline $\begin{array}{c}\text { Middle occipital gyrus } \\
\text { (right) }\end{array}$ & 34 & -88 & 14 & 6.84 & 165 & $.001^{1}$ \\
\hline $\begin{array}{c}\text { Middle occipital gyrus } \\
\text { (right) }\end{array}$ & 26 & -80 & 6 & 6.29 & $*$ & $.005^{1}$ \\
\hline $\begin{array}{c}\text { Middle occipital gyrus } \\
\text { (left) }\end{array}$ & -28 & -88 & 16 & 6.72 & 230 & $.002^{1}$ \\
\hline $\begin{array}{c}\text { Superior parietal } \\
\text { lobule (left) }\end{array}$ & -22 & -70 & 52 & 6.26 & $*$ & \\
\hline $\begin{array}{c}\text { Middle occipital gyrus } \\
\text { (left) }\end{array}$ & -26 & -92 & 2 & 6.05 & $* 05^{1}$ \\
\hline
\end{tabular}

${ }^{1} \mathrm{FWE}$-corrected to the entire brain volume; ${ }^{2} \mathrm{FWE}$-corrected to the volume of the ROI; ${ }^{*}$ Peak-level activation part of the above cluster 
Table 3. Main effects for the cognitive and emotional empathy tasks, for the stimuli type of neutral/emotional people, interaction effects of emotional/cognitive emotional task $x$ neutral/emotional people, and the main effects of things evaluation task.

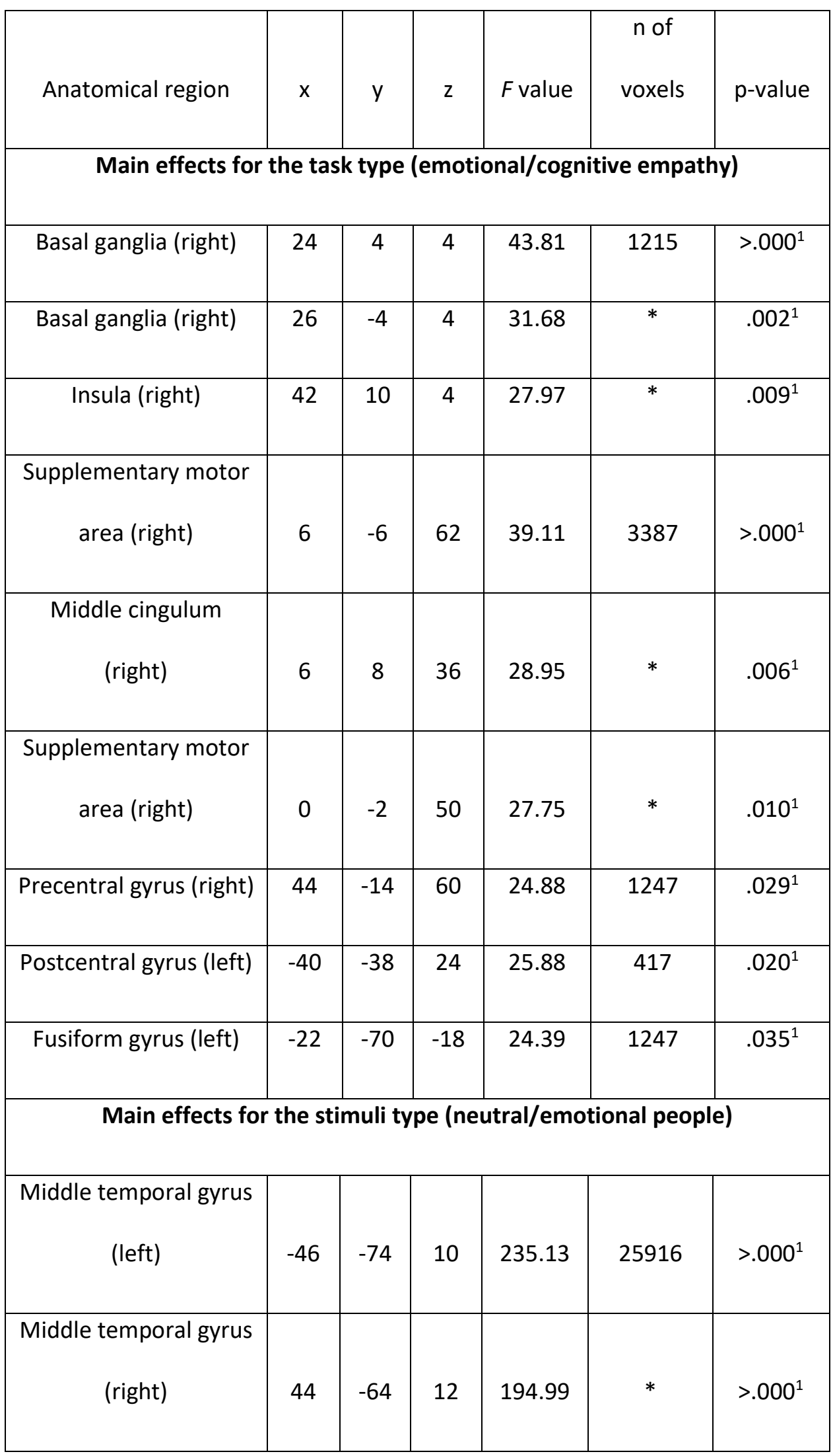




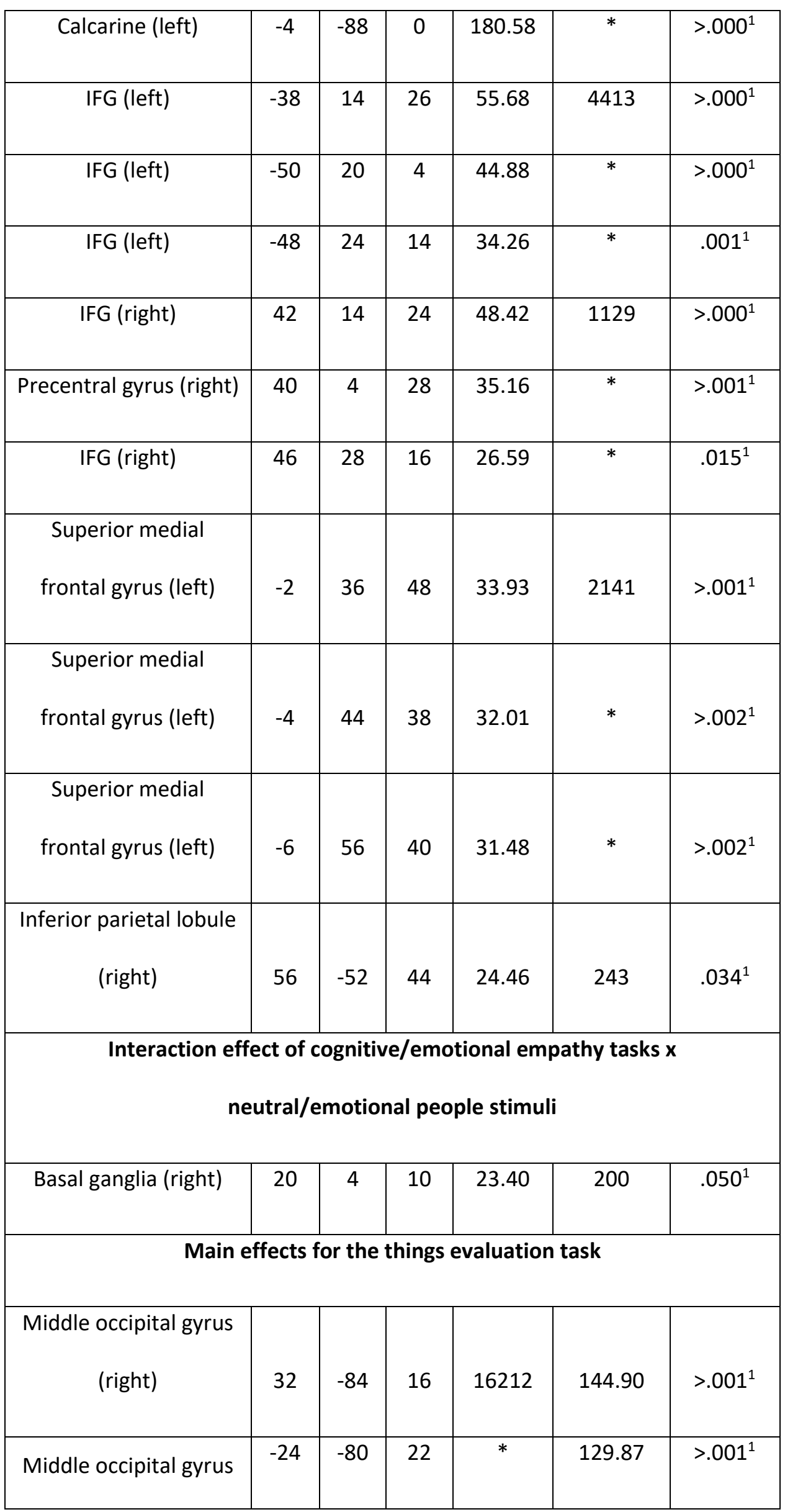




\begin{tabular}{|c|c|c|c|c|c|c|}
\hline (left) & & & & & & \\
\hline $\begin{array}{c}\text { Middle occipital gyrus } \\
\text { (left) }\end{array}$ & -28 & -94 & 20 & $*$ & 101.20 & $>.001^{1}$ \\
\hline $\begin{array}{c}\text { Medial frontal gyrus } \\
\text { (right) }\end{array}$ & 6 & 46 & 28 & 2155 & 57.81 & $>.001^{1}$ \\
\hline $\begin{array}{l}\text { Superior medial } \\
\text { frontal gyrus (left) }\end{array}$ & -2 & 46 & 30 & $*$ & 57.20 & $>.001^{1}$ \\
\hline Precuneus (right) & 4 & -60 & 36 & 1865 & 52.58 & $.001^{1}$ \\
\hline Precuneus (right) & 6 & -58 & 28 & $*$ & 51.11 & $.001^{1}$ \\
\hline Precuneus (right) & 6 & -50 & 24 & * & 47.01 & $.002^{1}$ \\
\hline $\begin{array}{c}\text { Middle temporal gyrus } \\
\text { (right) }\end{array}$ & 50 & -62 & 22 & 1965 & 44.66 & $.004^{1}$ \\
\hline Angural gyrus (right) & 48 & -52 & 30 & $*$ & 38.08 & $.014^{1}$ \\
\hline Angural gyrus (right) & 40 & -58 & 30 & $*$ & 37.32 & $.016^{1}$ \\
\hline Angural gyrus (left) & -50 & -64 & 28 & 1620 & 37.90 & $.014^{1}$ \\
\hline $\begin{array}{c}\text { Middle temporal gyrus } \\
\text { (left) }\end{array}$ & -48 & -58 & 22 & $*$ & 35.07 & $.026^{1}$ \\
\hline Angural gyrus (left) & -58 & -56 & 34 & $*$ & 32.34 & $.047^{1}$ \\
\hline IFG (right) & 44 & 18 & 22 & 800 & 36.52 & $.019^{1}$ \\
\hline IFG (left) & -30 & 8 & 28 & 451 & 35.62 & $.023^{1}$ \\
\hline
\end{tabular}

${ }^{1}$ FWE-corrected to the entire brain volume; ${ }^{2} \mathrm{FWE}$-corrected to the volume of the ROI; ${ }^{*}$ Peak-level activation part of the above cluster 
Table 4. Pairwise comparisons between the emotional empathy task > cognitive empathy task and emotional people during emotional empathy task > things evaluation task. The SQ score was modeled as a continuous covariate.

\begin{tabular}{|c|c|c|c|c|c|c|}
\hline Anatomical region & $\mathrm{x}$ & y & $z$ & $t$ value & $\begin{array}{c}\mathrm{n} \text { of } \\
\text { voxels }\end{array}$ & p-value \\
\hline \multicolumn{7}{|c|}{ Emotional empathy task > cognitive empathy task } \\
\hline Basal ganglia / insula & & & & & & \\
\hline (right) & 24 & 4 & 4 & 5.82 & 852 & $.014^{1}$ \\
\hline \multicolumn{7}{|c|}{ Emotional people during the emotional empathy task $>$ things evaluation } \\
\hline \multicolumn{7}{|c|}{ task } \\
\hline \multirow[t]{3}{*}{ PCC } & 2 & -56 & 20 & 8.75 & 2310 & $>.000^{1}$ \\
\hline & 2 & -58 & 28 & 7.48 & * & $>.000^{1}$ \\
\hline & 4 & -58 & 36 & 6.33 & $*$ & $.006^{1}$ \\
\hline mPFC (right) & 8 & 50 & 30 & 8.44 & 2533 & $>.000^{1}$ \\
\hline mPFC & 6 & 36 & 48 & 5.59 & $*$ & $.039^{1}$ \\
\hline STG (right) & 50 & -64 & 18 & 6.15 & 2493 & $>.000^{1}$ \\
\hline \multirow[t]{2}{*}{ STG (left) } & -48 & -66 & 26 & 5.13 & 78 & $>.000^{2}$ \\
\hline & -52 & -66 & 24 & 5.06 & $*$ & $.016^{2}$ \\
\hline IFG (right) & 44 & 20 & 26 & 6.82 & 1428 & $>.002^{1}$ \\
\hline IFG (right) & 34 & 30 & -10 & 5.90 & $*$ & $.018^{1}$ \\
\hline IFG (right) & 46 & 28 & -4 & 5.60 & * & $.039^{1}$ \\
\hline IFG (left) & -24 & 4 & 28 & 6.66 & 942 & $.002^{1}$ \\
\hline
\end{tabular}




\begin{tabular}{|c|c|c|c|c|c|c|}
\hline IFG (left) & -32 & 10 & 28 & 6.48 & $*$ & $.004^{1}$ \\
\hline Calcarine & -6 & -100 & 6 & 4.94 & 489 & $.015^{1}$ \\
\hline \\
Emotional people during emotional empathy task > things evaluation task, \\
SQ score as a covariate \\
\hline IFG (right) / insula & 26 & 24 & 16 & 6.36 & 465 & $.005^{1}$ \\
\hline
\end{tabular}

${ }^{1} \mathrm{FWE}$-corrected to the entire brain volume; ${ }^{2} \mathrm{FWE}$-corrected to the volume of the ROI; ${ }^{*}$ Peak-level activation part of the above cluster 
Figure legends

Figure 1. Activations during the spontaneous watching task. Upper row: The emotional people $>$ things comparison. The spontaneous watching of emotional pictures of people produced activations in areas commonly associated with social information processing including the MPFC, bilateral STG, and right IFGs, however, activations were not seen in the PCC ( $p=.001$ uncorrected for visualization). Lower row: Results of the regression analysis of the spontaneous watching task. In the emotional people > things comparison, the systemizing "brain type" (yellow) modeled as a covariate was associated with activations in the PCC (parameter estimates on the right from of the picture) and the left STG. High SQ scores (red) were associated with activations in the PCC, left STG, and right middle frontal gyrus. Systemizers thus had stronger activations during spontaneous watching in areas related to cognitive empathy. No activation differences were found in areas related to emotional empathy. The large activation cluster seen in the mPFC did not survive FWE-correction at the voxel-wise level to the whole brain $(p=.280$; cluster-wise correction $p=.006$, cluster extend 457). $p=.005$ uncorrected for visualization

Figure 2. Upper row: Main effects of the cognitive/emotional empathy tasks. Main effects for the empathy tasks were seen in the fusiform gyrus, precentral gyrus and especially in the supplementary motor area and in a large cluster in the right basal ganglia. The basal ganglia activations were related to stronger activation for emotional empathy task. Lower row picture A: In the pairwise comparison between emotional people stimuli during emotional empathy task and things evaluation task, low SQ score was associated with higher activation in the insula/right IFG. In other words, empathizers had stronger activation in areas commonly associated with emotional empathy. Lower row picture B: there was also an interaction effect between emotional empathy task and pictures of emotional people in the right basal ganglia. $p=.001$ uncorrected for visualization 